\title{
Deep reflection-mode photoacoustic imaging of internal organs
}

Kwang Hyun Song, Lihong V. Wang

Kwang Hyun Song, Lihong V. Wang, "Deep reflection-mode photoacoustic imaging of internal organs," Proc. SPIE 6856, Photons Plus Ultrasound: Imaging and Sensing 2008: The Ninth Conference on Biomedical Thermoacoustics, Optoacoustics, and Acousto-optics, 68561W (28 February 2008); doi: 10.1117/12.761389

SPIE. Event: SPIE BiOS, 2008, San Jose, California, United States 


\title{
Deep reflection-mode photoacoustic imaging of internal organs
}

\author{
Kwang Hyun Song and Lihong V. Wang \\ Optical Imaging Laboratory, Department of Biomedical Engineering, \\ Washington University in Saint Louis
}

\begin{abstract}
A deep reflection-mode photoacoustic imaging system was developed and demonstrated to possess a maximum imaging depth up to $38 \mathrm{~mm}$ in chicken breast tissue. Using this system, structures in the thoracic cavity and vasculature in cervical area of rats were clearly imaged. Particularly, part of the heart was imaged. In the thoracic cavity, the right atrium imaged, which is one of deepest, was situated $\sim 7 \mathrm{~mm}$ deep. In the cervical area, common carotid artery and jugular vein were imaged, which are appropriate for the study of oxygenation between artery and vein. In the abdominal cavity, the embedded structures of a kidney, spinal cord, and vena cava inferior were also clearly imaged in situ and in vivo. The depth of the vena cava inferior was as deep as $\sim 15 \mathrm{~mm}$ in vivo. This study shows the depth capability of the system in animals. This imaging modality can be a useful tool to diagnose the disease of organs by assessing the morphological and functional changes in the blood vessels and the organs.
\end{abstract}

Keywords: Photoacoustic imaging, internal organ, thoracic cavity, cervical, kidney

\section{INTRODUCTION}

In the medical imaging, magnetic resonance imaging (MRI) and computed tomography (CT) have played an important role to diagnose cancer in internal organs by mostly inspecting the morphological changes (1). Recently, with an aid of contrast agents, those imaging methods can provide functional information like oxygenation in veins, blood flow, and blood volume $(2,3)$. Most importantly, they come to have an improved sensitivity (4-6). However, both imaging modalities are quite expensive and have a long acquisition time. In particular, CT is ionizing method and can cause cancer (7). In addition, without contrast agent, both imaging modalities have less sensitivity and reliability to detect early cancer $(5,6,8-10)$. In early stage cancer, since angiogenesis is more dominant than morphological changes, optical imaging modality, which is highly sensitive to intrinsic optical contrast like hemoglobin, have a strong advantage over MRI and CT. Furthermore, it is capable of functional imaging, such as blood volume and oxygenation (11-13). But, purely optical imaging modalities have poor spatial resolution at deeper regions because of the overwhelming optical scattering in soft tissues.

Photoacoustic imaging modality is an emerging and advancing technology in biomedical imaging overcoming the poor spatial resolution of purely optical imaging methods. Since photoacoustic imaging technology employs a pulsed laser light for the photoacoustic excitation, it is non-ionizing, safe, and highly sensitivity to optical contrast including endogenous and exogenous. In addition, this imaging technology has scalable ultrasonic resolutions (14-16) and provides good imaging depth $\sim 5 \mathrm{~cm}$ in biological tissue (17) since it receives ultrasonic waves as the photoacoustic signals, which have much less scattering than optical scattering in soft tissues. Furthermore, it is relatively inexpensive method. With these fundamental advantages, this technology has been widely applied to the small animal imaging, such as vasculature imaging in skin (18-20), brain cortex imaging (21), and molecular targeted imaging (22). In this imaging technique, there are latest advances. Photoacoustic microscopy was developed in the reflection mode (23), and has produced plenty of remarkable structural and functional images in the skin $(20,24)$. This was proven to be more versatile to image various objects. With the same illumination geometry, optical detection based photoacoustic imaging system was also developed and has showed the vasculature images in the skin and the cortical surface of the mice brain (25). But, the imaging depths of both systems were limited to $\sim 3 \mathrm{~mm}$ since they used low optical fluence for the photoacoustic excitation and higher central frequency ultrasonic detectors. To exceed this limited depth, the deep reflection-mode photoacoustic

Photons Plus Ultrasound: Imaging and Sensing 2008: The Ninth Conference on Biomedical

Thermoacoustics, Optoacoustics, and Acousto-optics, edited by Alexander A. Oraevsky, Lihong V. Wang,

Proc. of SPIE Vol. 6856, 68561W, (2008) $\cdot 1605-7422 / 08 / \$ 18 \cdot$ doi: 10.1117/12.761389 
imaging system was developed to image deeper structures and showed the preliminary organ images of a relative large rat in author's previous work (26). Even though it demonstrated $\sim 38 \mathrm{~mm}$ imaging depth by imaging a horse hair in chicken breast tissue, the preliminary images just showed the shallowly located organs. In particular, it didn't show the internal structure of them either because of the high optical absorption of them. When an imaging object has higher optical absorption, photoacoustic signal is mostly generated on the surface of the object. This is the well-known challenge of the photoacoustic imaging. However, there are still plenty of organs that we can image according to their depth, location, configuration, and absorption.

As the follow up study toward clinical application, we imaged various organs such as structures in the thoracic cavity and vasculatures in the cervical area of a rat. In the abdominal cavity, the embedded structures of a kidney were clearly imaged. In this study, we also show the depth capability of this imaging modality in small animals. We expect that this imaging technique can be a useful tool to diagnose diseases in organs by inspecting its structural changes. Especially, this will be good complement to current diagnosing imaging methods to detect early cancer based on their morphological and functional changes.

\section{METHOD AND MATERIAL}

\subsection{Photoacoustic imaging system}

The schematic of the deep reflection-mode photoacoustic imaging system is shown in Figure 1. The main configurations of this system are a light illumination, a raster scanning, and a data acquisition. The light illumination employs a darkfield ring-shaped illumination instead of a bright-field one. This illumination can give an efficient detection of the photoacoustic signals generated from deeper regions because of the reduced photoacoustic signal generation on the surface. The surface signals can interfere with those from deeper region and obscure the detection of deep signals. This illumination is formed by a concave lens, a spherical conical lens, and an optical condenser attached in an optical rail. To accomplish deeper imaging, it is important to deliver the higher optical energy within the maximum permissible exposure limitation (MPE: $31 \mathrm{~mJ} / \mathrm{cm}^{2}$ at $800 \mathrm{~nm}$ ). Therefore, we used two prisms to deliver the laser light instead of an optical fiber, and broadened the beam sufficiently.

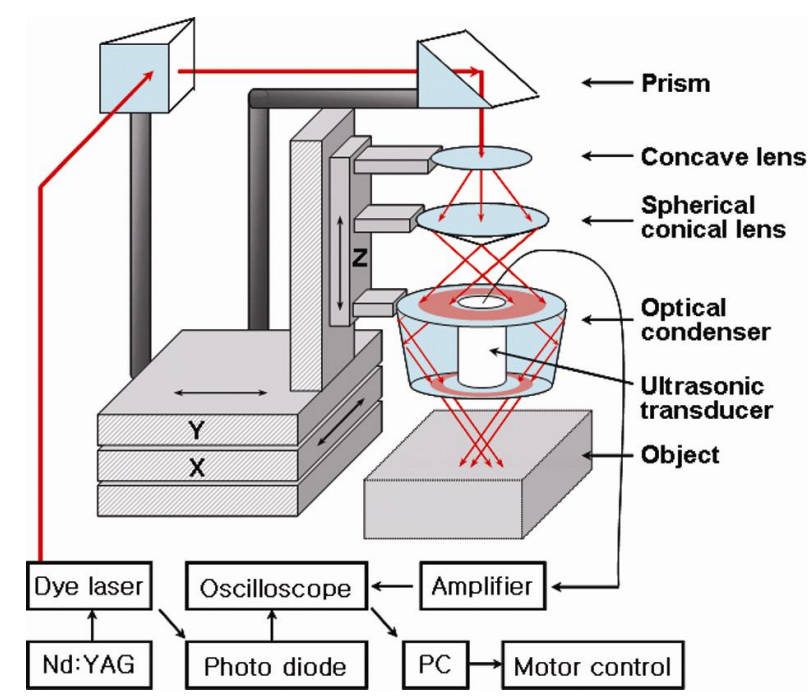

Figure 1: The schematic of the deep reflection-mode photoacoustic imaging system employing the dark-field illumination. The optical part is for the dark-field and donut-shape illumination and the mechanical part is for the raster scanning. The signals received by an ultrasonic transducer are digitized by an oscilloscope and collected by a computer.

To penetrate deeper in biological tissue, $800-\mathrm{nm}$ optical wavelength was chosen in the near-infrared range for the photoacoustic excitation. This wavelength is suitable for structural image because it is isosbestic point of molar 
extinction spectra of oxy- and deoxy-hemoglobin and insensitive oxygenation of the target. The light source was a tunable $(720 \mathrm{~nm}-830 \mathrm{~nm}$ ) Ti:sapphire laser (LT-2211A, LOTIS TII) pumped by a Q-switched Nd:YAG laser (LS$2137 / 2$, LOTIS TII). Light pulses from this laser have a $<15$-ns pulse duration with a $10-\mathrm{Hz}$ pulse-repetition frequency. Since the laser system has pulse-to-pulse fluctuations in energy, the energy of each pulse was measured by a photodiode (DET110, Thorlabs) and used for compensating the corresponding photoacoustic signal.

For the photoacoustic signal detection, a $3.5-\mathrm{MHz}$ or a $5-\mathrm{MHz}$ central frequency ultrasonic transducer (V380, V308, Panametrics-NDT) was employed since lower frequency of ultrasound has less attenuation than higher one. We chose the spherically focused ultrasonic transducer since we employed the X-Y raster scanning mechanism to obtain 3-D data. Both transducers have a $4.95-\mathrm{cm}$ and a $2.54-\mathrm{cm}$ focal length, a $2.54-\mathrm{cm}$ and a $1.91-\mathrm{cm}$ diameter active element, and a $70 \%$ and a $72 \%$ nominal bandwidth, respectively. The f-numbers (focal length / diameter of active element) of them are 1.95 and 1.33 , respectively. The $3.5-\mathrm{MHz}$ central frequency ultrasonic transducer is good for deeper imaging since it has a longer focal length, and the 5-MHz one is suited to the high resolution imaging since it has lower f-number providing a better transverse resolution. The transducer was immersed in a water container, and scanned the object through a $5 \mathrm{~cm} \mathrm{x}$ $5 \mathrm{~cm}$ opening sealed with a thin clear membrane. For acoustic coupling between the object and the clear membrane, ultrasonic gel was used (Ultrasound Scanning Gel, Sonotech, Inc). The axial and the transverse resolution were $\sim 144 \mu \mathrm{m}$ and $\sim 560 \mu \mathrm{m}$ at $19 \mathrm{~mm}$ in $10 \%$ gelatin phantom containing $1 \%$ intralipid with the $5-\mathrm{MHz}$ central frequency ultrasonic transducer (26). The SNR was measured to be $27 \mathrm{~dB}$ at $19 \mathrm{~mm}$ in the same gelatin phantom.

The raster scanning was performed to obtain 3-D data using a XY linear translation stage (XY-6060, Danaher Motion), which was motorized and controlled by a computer. In the data acquisition, a continuous scanning was employed without signal averaging to shorten the acquisition time. The raster scanning and the data acquisition were synchronized with the photodiode signal, which was also used for the energy compensation. The PA signals from the object were first amplified by an amplifier (5072PR, Panametrics-NDT), and digitized by an oscilloscope (Tektronix TDS 5054) with $50 \mathrm{M}$ sampling rate.

\subsection{Animal}

For internal organ imaging, Sprague Dawley rats weighing $120 \sim 390 \mathrm{~g}$ were used. The rat was initially anesthetized with a mixture of Ketamine $(85 \mathrm{mg} / \mathrm{kg})$ and Xylazine $(15 \mathrm{mg} / \mathrm{kg})$ for the hair removal procedure. The hair on the region of interest was gently removed using a commercial hair-removal lotion before imaging. For an in situ experiment, the animal was euthanized by pentobarbital overdose. For an in vivo experiment, the animal was kept under the anesthesia using a vaporized isoflurane anesthesia system $(1 \mathrm{~L} / \mathrm{min}$ oxygen and $0.75 \%$ isoflurane, Euthanex Corp.) during all image acquisitions. Both $\mathrm{PSO}_{2}$ and the rate of heart were monitored using a pulseoximeter (NONIN Medical INC., 8600V). During image acquisitions, $8 \mathrm{ml}$ of $0.9 \%$ saline was administered for hydration. After image acquisitions, the animal was also euthanized by pentobarbital overdose. All animal experiments were conducted in compliance with the guideline on the care and the use of laboratory animals from Washington University in St. Louis.

\section{RESULT AND DISCUSSION}

\subsection{Photoacoustic vasculature imaging of the thoracic cavity and cervical area of a rat}

As the application of the deep reflection-mode photoacoustic imaging system, the structure in the thoracic cavity and the vasculature in the cervical area of a rat weighing $\sim 400 \mathrm{~g}$ were imaged. The rat was laid down on the animal holder with the chest upward. Forepaws were gently stretched out. Figure 2A is the non-invasive photoacoustic maximum amplitude projection (MAP) image of the thoracic cavity and the cervical area in situ. The structures are indicated with arrows and abbreviations: RC. Right common carotid artery, RE. Right external jugular vein, RI. Right internal Jugular vein, RA. Right atrium, BV. Blood vessels on the heart surface, BS. Blood vessel on sternum, ST. Sternum. Figure. 2B is the corresponding anatomical photograph of the thoracic cavity and cervical area taken after obtaining the PA image. Figure $2 \mathrm{C}$ shows the blood vessels around ribs. Figure 2A shows the same blood vessels as those in Figure $2 \mathrm{~B}$ and $2 \mathrm{C}$. Photoacoustic B-Scan images are shown in Figure 2D, obtained at the dotted lines in Figure 2A. These B-Scan images show the depth of each structure. Right common carotid artery was situated at around $6 \mathrm{~mm}$, and right atrium was located at around $7 \mathrm{~mm}$. 


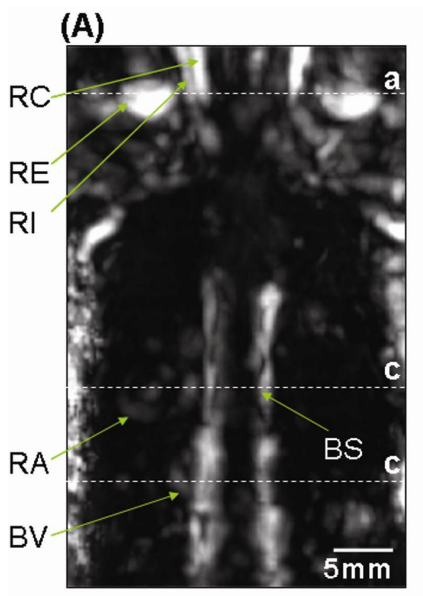

(B)

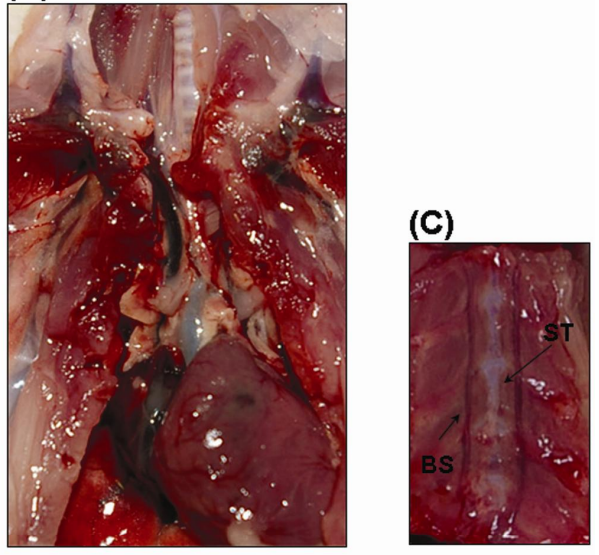

(D)
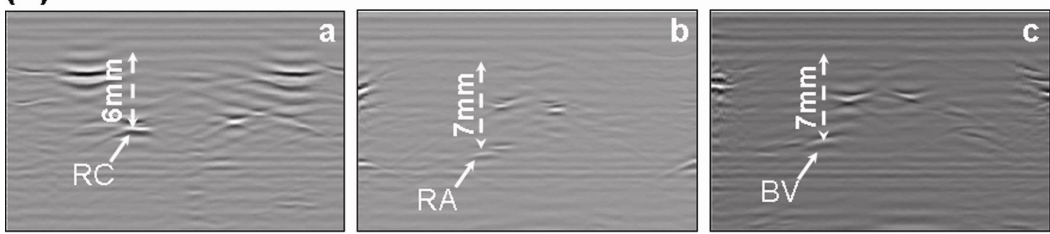

Figure 2. Non-invasive photoacoustic imaging of the thoracic and the cervical area of a rat in situ. A, Photoacoustic MAP image. B, Corresponding anatomical photograph after imaging. C, Photograph of the rib cage flipped over. D, Photoacoustic B-Scan images corresponding to a, b, and c in Figure 2A. Structures: RC. Right common carotid artery, RE. Right external jugular vein, RI. Right internal Jugular vein, RA. Right atrium, BV. Blood vessels on the heart surface, BS. Blood vessel on sternum, ST. Sternum..

As shown in Figure 2C, the thoracic cavity was caged with the ribs and the sternum. The sternum is located right above the heart. With this reason and employing the raster scanning based on a single ultrasonic transducer, this imaging system has small windows where the ultrasonic transducer receive the PA signal from the internal structures through ribs. Therefore, in Figure 2A, heart and other structures were not clearly imaged.

This structural image can give the information about the disease on blood vessels in the thoracic cavity even on the heart by inspecting the structural changes in them. Besides this structural information, this imaging can also provide the functional information like oxygen saturation and blood volume change to the endogenous and/or exogenous changes. Along with the small windows between ribs, breathing motion is another challenge in organ imaging in vivo. This irregular breathing changes the positions of internal organs including blood vessels and intestines. Inhale and exhale gating imaging can be a solution for this breathing motion. In particular, with those gating, a real-time ultrasonic array system will be the best solution for the breathing. In addition, this array system will receive the better photoacoustic signals through the small window than a raster scanning system.

\subsection{Photoacoustic kidney imaging of a rat in situ}

Another good application suitable for this imaging system is the kidney imaging of small animals. The major function of it is to remove wastes from the blood stream and excrete them. Also, it is deeply involved in the body homeostasis. The kidney of a rat weighing $\sim 120 \mathrm{~g}$ was imaged noninvasively in situ with the same imaging set up. Figure $3 \mathrm{~A}$ is noninvasive photoacoustic saggital MAP image of the rat, showing the structures within the skin and the top surface of the kidney. Since the photoacoustic signal is depth-resolved, the MAP image can be constructed within a certain depths. 


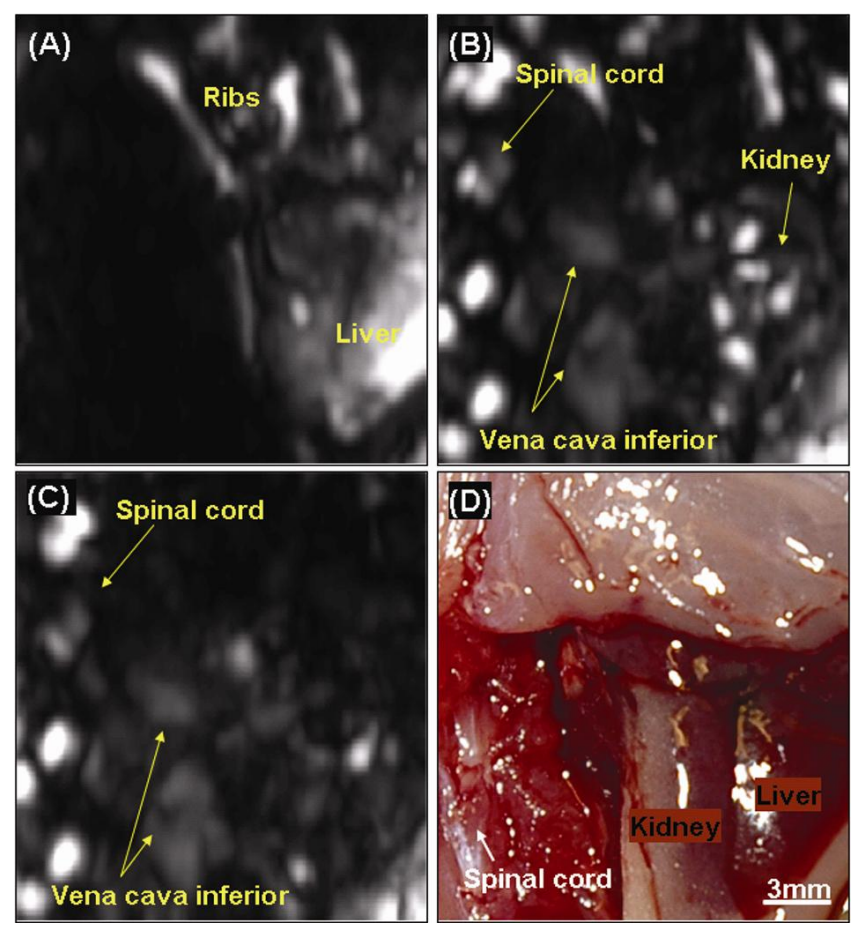

Figure 3. Non-invasive photoacoustic image of the kidney of the rat. A, Saggital MAP image of the structures between the skin and the top surface of the kidney. B, Saggital MAP image of the kidney, the spinal cord, and the vena cava inferior. C, Saggital MAP image below the kidney, showing the vena cava inferior only. D, Corresponding anatomical photograph with ribs intact.

Figure 3A shows the blood vessels around the ribs and some parts of the liver. Those are well matched with anatomical photograph in Figure 3D. Figure 3B is the saggital MAP image formed with the rest of the same data, which shows the kidney, the spinal cord, and the vena cava inferior. In particular, the interlobar arteries of the kidney were clearly imaged. Those three structures are also in good match with the corresponding anatomical photograph in Figure 3D. To see the vena cava inferior more clearly, another saggital MAP image was constructed with the photoacoustic data below the kidney (Figure 3C). Figure 3C shows the vena cava inferior better excluding other features. Figure 4 is the axial MAP image, showing the depths of all structures. The kidney was located $\sim 6 \mathrm{~mm}$, the spinal cord was situated at $\sim 5.5 \mathrm{~mm}$, and the vena cava inferior is positioned at $\sim 10 \mathrm{~mm}$.

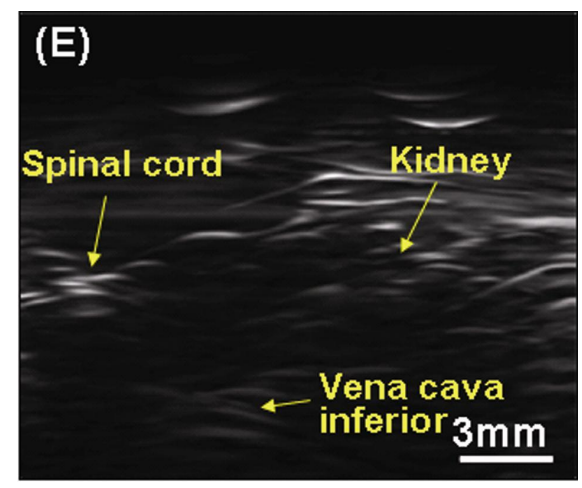

Figure 4. Axial MAP image of the same rat as in Figure 3, showing the depths. 


\subsection{Non-invasive photoacoustic kidney imaging of a rat in vivo}

The breathing motion is again the challenge for this kidney imaging in vivo. This breathing changes the position of organs and interrupts the efficient photoacoustic signal generation. Despite of the breathing motion, we can still receive the photoacoustic signals. We acquired in vivo photoacoustic kidney image non-invasively (Figure 5A). Kidney and vena cava inferior were imaged. Spinal cord was out of field of view. Figure 5B is the corresponding anatomical photograph. Figure $5 \mathrm{C}$ is one of B-Scan images, showing the depth of the vena cava inferior. The depth of this blood vessel was $\sim 15$ $\mathrm{mm}$. This study shows the depth capability of the imaging system in the animal model, which has higher absorption and more heterogeneity than chicken breast tissue. It is also expected to be helpful to understand the function of the kidney.
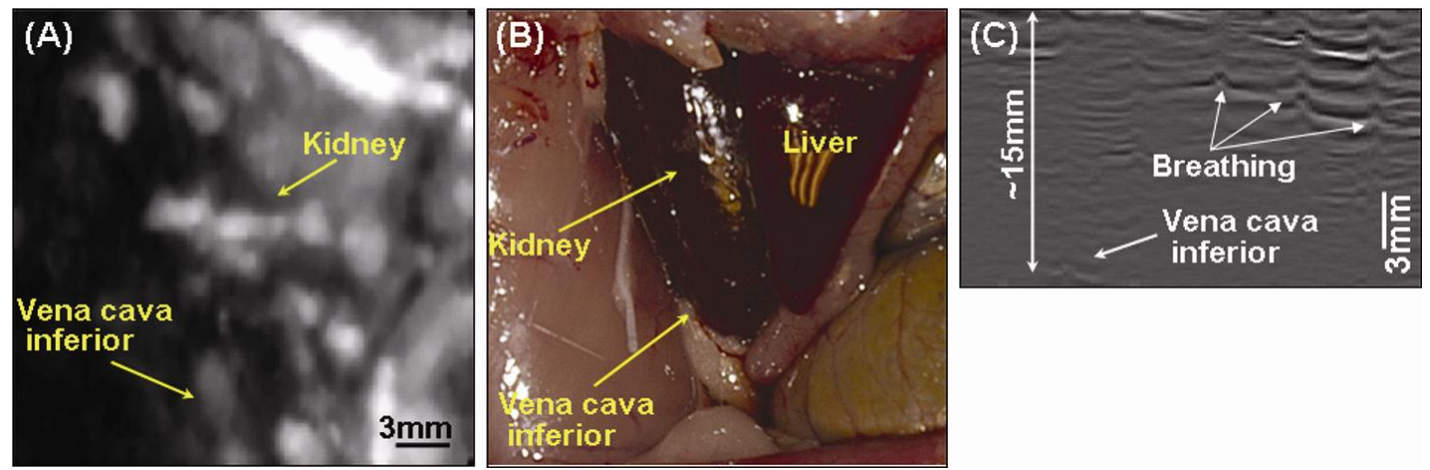

Figure 5. Non-invasive photoacoustic in vivo kidney image of a rat. A, Saggital MAP image. B, Corresponding anatomical photograph. C, Photoacoustic B-Scan image, showing the depth of vena cava inferior and breathing motion.

\section{CONCLUSION}

We successfully imaged the thoracic cavity and the cervical area of a rat using the reflection-mode photoacoustic imaging system. The part of the heart and right atrium were imaged in the thoracic cavity. The common carotid artery and jugular vein were also imaged. Whole heart was not clearly imaged since it was caged with the ribs and the sternum. Thus, this imaging system has small window to receive the PA signals from the heart. For abdominal imaging, we clearly imaged the interlobar arteries, the vena cava inferior, and the spinal cord in situ. Since in situ imaging has no breathing motion, every structure was clearly visualized. But, in vivo imaging didn't produce as clear structures as in situ because it has the breathing motion. We also show the depth capability of the imaging system in the animal model. This study lays the foundation of the internal organ imaging in the photoacoustic imaging. In addition, this imaging technique can be a useful tool to study the abnormality and function of the organs toward clinical application

\section{ACKNOWLEDGEMENT}

We thank Konstantine Maslov for fruitful discussion and assistance. The project sponsored by National Institutes of Health grants R01 CA106728 and R01 NS46214 (BRP). L. Wang's email is lhwang@,biomed.wustl.edu. 


\section{REFERENCES}

1. G. Antoch, F. M. Vogt, L. S. Freudenberg, F. Nazaradeh, S. C. Goehde, J. Barkhausen, G. Dahmen, A. Bockisch, J. F. Debatin and S. G. Ruehm, "Whole-body dual-modality PET/CT and whole-body MRI for tumor staging in oncology," Jama-J Am Med Assoc 290(24), 3199-3206 (2003)

2. P. V. Prasad, A. Priatna, K. Spokes and F. H. Epstein, "Changes in intrarenal oxygenation as evaluated by BOLD MRI in a rat kidney model for radiocontrast nephropathy," Journal of Magnetic Resonance Imaging 13(5), 744-747 (2001)

3. T. G. Purdie, E. Henderson and T. Y. Lee, "Functional CT imaging of angiogenesis in rabbit VX2 soft-tissue tumour," Physics in medicine and biology 46(12), 3161-3175 (2001)

4. S. C. Rankin, "MRI of the breast," The British journal of radiology 73(872), 806-818 (2000)

5. E. Kresnik, P. Mikosch, H. J. Gallowitsch, D. Kogler, S. Wieser, M. Heinisch, O. Unterweger, W. Raunik, G. Kumnig, I. Gomez, G. Grunbacher and P. Lind, "Evaluation of head and neck cancer with F-18-FDG PET: a comparison with conventional methods," European Journal of Nuclear Medicine 28(7), 816-821 (2001)

6. B. Nowak, E. Di Martino, S. Janicke, U. Cremerius, G. Adam, M. Zimny, P. Reinartz and U. Bull, "Diagnostic evaluation of malignant head and neck cancer by F-18-FDG PET compared to CT/MRI," Nuklearmedizin-Nuclear Medicine 38(8), 312-318 (1999)

7. K. Nishizawa, T. Maruyama, M. Takayama, M. Okada, J. Hachiya and Y. Furuya, "Determinations of organ doses and effective dose equivalents from computed tomographic examination," The British journal of radiology 64(757), 20-28 (1991)

8. P. L. Davis and K. S. McCarty, "Sensitivity of enhanced MRI for the detection of breast cancer: new, multicentric, residual, and recurrent," European radiology 7(S289-S298 (1997)

9. L. D. Buadu, J. Murakami, S. Murayama, N. Hashiguchi, S. Sakai, K. Masuda, S. Toyoshima, S. Kuroki and S. Ohno, "Breast lesions: correlation of contrast medium enhancement patterns on MR images with histopathologic findings and tumor angiogenesis," Radiology 200(3), 639-649 (1996)

10. B. Levin, D. Brooks, R. A. Smith and A. Stone, "Emerging technologies in screening for colorectal cancer: CT colonography, immunochemical fecal occult blood tests, and stool screening using molecular markers," CA: a cancer journal for clinicians 53(1), 44-55 (2003)

11. D. A. Benaron, S. R. Hintz, A. Villringer, D. Boas, A. Kleinschmidt, J. Frahm, C. Hirth, H. Obrig, J. C. van Houten, E. L. Kermit, W. F. Cheong and D. K. Stevenson, "Noninvasive functional imaging of human brain using light," J Cereb Blood Flow Metab 20(3), 469-477 (2000)

12. J. E. Brazy, D. V. Lewis, M. H. Mitnick and F. F. Jobsis vander Vliet, "Noninvasive monitoring of cerebral oxygenation in preterm infants: preliminary observations," Pediatrics 75(2), 217-225 (1985)

13. B. Chance, E. Anday, S. Nioka, S. Zhou, L. Hong, K. Worden, C. Li, T. Murray, Y. Ovetsky, D. Pidikiti and R. Thomas, "A novel method for fast imaging of brain function, non-invasively, with light," Opt Express 2(10), 411$423(1998)$

14. C. G. A. Hoelen, F. F. M. de Mul, R. Pongers and A. Dekker, "Three-dimensional photoacoustic imaging of blood vessels in tissue," Optics letters 23(8), 648-650 (1998)

15. R. A. Kruger, W. L. Kiser, Jr., D. R. Reinecke and G. A. Kruger, "Thermoacoustic computed tomography using a conventional linear transducer array," Medical physics 30(5), 856-860 (2003)

16. V. Ntziachristos, J. Ripoll, L. V. Wang and R. Weissleder, "Looking and listening to light: the evolution of wholebody photonic imaging," Nature biotechnology 23(3), 313-320 (2005)

17. G. Ku and L. V. Wang, "Deeply penetrating photoacoustic tomography in biological tissues enhanced with an optical contrast agent," Optics letters 30(5), 507-509 (2005)

18. R. G. M. Kolkman, E. Hondebrink, W. Steenbergen and F. F. M. de Mul, "In vivo photoacoustic imaging of blood vessels using an extreme-narrow aperture sensor," Ieee J Sel Top Quant 9(2), 343-346 (2003)

19. R. J. Zemp, R. Bitton, M. L. Li, K. K. Shung, G. Stoica and L. V. Wang, "Photoacoustic imaging of the microvasculature with a high-frequency ultrasound array transducer," Journal of biomedical optics 12(1), 010501 (2007)

20. H. F. Zhang, K. Maslov, M. L. Li, G. Stoica and L. H. V. Wang, "In vivo volumetric imaging of subcutaneous microvasculature by photoacoustic microscopy," Opt Express 14(20), 9317-9323 (2006)

21. X. Wang, Y. Pang, G. Ku, X. Xie, G. Stoica and L. V. Wang, "Noninvasive laser-induced photoacoustic tomography for structural and functional in vivo imaging of the brain," Nature biotechnology 21(7), 803-806 (2003) 
22. M.-L. Li, J. Oh, X. Xie, G. Ku, W. Wang, C. Li, G. Lungu, G. Stoica and L. V. Wang, "Simultaneous molecular and hypoxia imaging of brain tumors in vivo using spectroscopic photoacoustic tomography," Proceedings of IEEE (2007)

23. K. Maslov, G. Stoica and L. V. Wang, "In vivo dark-field reflection-mode photoacoustic microscopy," Optics letters 30(6), 625-627 (2005)

24. H. F. Zhang, K. Maslov, G. Stoica and L. H. V. Wang, "Functional photoacoustic microscopy for high-resolution and noninvasive in vivo imaging," Nature biotechnology 24(7), 848-851 (2006)

25. E. Z. Zhang, L. Jan and B. Paul, "Three dimensional photoacoustic imaging of vascular anatomy in small animals using an optical detection system," Proc SPIE 6437(64370S-1), (2007)

26. K. H. Song and L. V. Wang, "Deep reflection-mode photoacoustic imaging of biological tissue," Journal of biomedical optics 12(6), 060503 (2007) 